\title{
The Novel Phosphodiesterase 9A Inhibitor BI 409306 Increases Cyclic Guanosine Monophosphate Levels in the Brain, Promotes Synaptic Plasticity, and Enhances Memory Function in Rodents $\underline{[}$
}

\author{
Holger Rosenbrock, Riccardo Giovannini, Gerhard Schänzle, Eliza Koros, Frank Runge, \\ Holger Fuchs, Anelise Marti, ${ }^{1}$ Klaus G. Reymann, Ulrich H. Schröder, Ernesto Fedele, \\ and Cornelia Dorner-Ciossek
}

\begin{abstract}
Boehringer Ingelheim International GmbH, Biberach an der Riss, Germany (H.R., R.G., G.S., E.K., F.R., H.F., A.M., C.D.-C.); Leibniz Institute for Neurobiology, Magdeburg, Germany (K.G.R., U.H.S.); and Department of Pharmacy, Centre of Excellence for Biomedical Research, University of Genova, Genova, Italy (E.F.)
\end{abstract}

Received May 15, 2019; accepted September 25, 2019

\begin{abstract}
$N$-methyl-D-aspartate (NMDA) receptor-dependent long-term potentiation (LTP) is an established cellular model underlying learning and memory, and involves intracellular signaling mediated by the second messenger cyclic guanosine monophosphate (cGMP). As phosphodiesterase (PDE)9A selectively hydrolyses CGMP in areas of the brain related to cognition, PDE9A inhibitors may improve cognitive function by enhancing NMDA receptor-dependent LTP. This study aimed to pharmacologically characterize BI 409306, a novel PDE9A inhibitor, using in vitro assays and in vivo determination of cGMP levels in the brain. Further, the effects of BI 409306 on synaptic plasticity evaluated by LTP in ex vivo hippocampal slices and on cognitive performance in rodents were also investigated. In vitro assays demonstrated that $\mathrm{BI} 409306$ is a potent and selective inhibitor of human and rat PDE9A with mean concentrations at half-maximal inhibition $\left(\mathrm{IC}_{50}\right)$ of 65 and $168 \mathrm{nM}$. BI 409306 increased cGMP levels in rat prefrontal cortex and cerebrospinal fluid and attenuated a reduction in mouse striatum cGMP induced by the NMDA-receptor antagonist MK-801. In ex vivo rat brain slices, BI 409306 enhanced
\end{abstract}

\section{Introduction}

Cognitive function, memory, and learning are closely associated with glutamatergic neurotransmission, with the postsynaptic $N$-methyl-D-aspartate (NMDA) receptor playing an

This work was funded by Boehringer Ingelheim GmbH. The sponsor was given the opportunity to review the manuscript for medical and scientific accuracy as well as intellectual property considerations. Primary laboratory of origin: Boehringer Ingelheim International $\mathrm{GmbH}$, Biberach an der Riss, Germany. Giovannini was the inventor of BI 409306.

${ }^{1}$ Current affiliation: Teva Pharmaceutical International $\mathrm{GmbH}$, Basel, Switzerland.

Parts of this work have been published previously in abstract form: DornerCiossek C, Giovannini R, and Rosenbrock H (2015) BI 409306, a nove phosphodiesterase 9A inhibitor, part I: potency, selectivity and in-vitro functional characterization on synaptic plasticity. Schizophr Bull 41(Suppl 1):S31; Rosenbrock H, Marti A, Koros E, Runge F, Fuchs H, Giovannini R, and Dorner-Ciossek C (2015) BI 409306, a novel phosphodiesterase 9A inhibitor, part II: in-vivo characterization regarding target engagement and cognition tasks in rodents. Schizophr Bull 41(Suppl 1):S36.

https://doi.org/10.1124/jpet.119.260059.

S This article has supplemental material available at jpet.aspetjournals.org.
LTP induced by both weak and strong tetanic stimulation. Treatment of mice with $\mathrm{Bl} 409306$ reversed MK-801-induced working memory deficits in a T-maze spontaneous-alternation task and improved long-term memory in an object recognition task. These findings suggest that $\mathrm{BI} 409306$ is a potent and selective inhibitor of PDE9A. BI 409306 shows target engagement by increasing cGMP levels in brain, facilitates synaptic plasticity as demonstrated by enhancement of hippocampal LTP, and improves episodic and working memory function in rodents.

\section{SIGNIFICANCE STATEMENT}

This preclinical study demonstrates that $\mathrm{BI} 409306$ is a potent and selective PDE9A inhibitor in rodents. Treatment with $\mathrm{BI}$ 409306 increased brain cGMP levels, promoted long-term potentiation, and improved episodic and working memory performance in rodents. These findings support a role for PDE9A in synaptic plasticity and cognition. The potential benefits of $\mathrm{Bl} 409306$ are currently being investigated in clinical trials. (Fisher et al, 1998). Furthermore, inhibition of PDE9A in brain tissue leads to an increase in cGMP, suggesting a role in regulation of basal levels of cGMP in the brain (Kleiman et al., 2012). As PDE9A 
is expressed in neurons of brain regions related to cognition, such as the neocortex and hippocampus (Andreeva et al., 2001), this enzyme isoform is well placed to regulate NMDA receptor signaling pathways associated with synaptic plasticity and cognitive function (Reneerkens et al., 2009; Kroker et al., 2012; Dorner-Ciossek et al., 2017). In support of this hypothesis, preclinical studies have shown that inhibition of PDE9A can increase cGMP levels in the rat brain, enhance LTP, and improve memory function in rodent cognition tasks (van der Staay et al., 2008; Hutson et al., 2011; Kleiman et al., 2012; Kroker et al., 2012, 2014). In addition, a dose-dependent increase in cGMP levels in cerebrospinal fluid (CSF) of healthy volunteers has been demonstrated following a single oral administration of the PDE9A inhibitor BI 409306 (Boland et al., 2017).

BI 409306 is a novel, potent, and selective PDE9A inhibitor in clinical development (Moschetti et al., 2016; Boland et al., 2017; Brown et al., 2019; Frölich et al., 2019). The current study aimed to assess the in vitro potency and selectivity of BI 409306 and to evaluate its functional target engagement in vivo through measurements of cGMP levels in brain tissue and CSF. Furthermore, the effects of BI 406309 on synaptic stabilization and plasticity were assessed through electrophysiological recordings of long-term potentiation in rat hippocampal slices. The efficacy of BI 409306 on cognition was also evaluated in tasks assessing working and episodic memory in mice.

\section{Materials and Methods}

\section{Drugs}

The structure of BI 409306 is shown in Fig. 1. BI 409306 was synthesized at Boehringer Ingelheim Italia, Chemistry Research Center (Milan, Italy). MK-801 as hydrogen maleate or hydrochloride salt was obtained from Sigma Aldrich (Saint-Quentin Fallavier, France, and Taufkirchen, Germany, respectively).

\section{Determination of In Vitro Potency and Selectivity of BI 409306}

Cell extracts containing PDE isoforms were produced using a baculovirus expression system (Bac-To-Bac; Invitrogen, Carlsbad, $\mathrm{CA})$. The open reading frames of PDEs were cloned into the plasmid vector pFastbac (human PDE2A, 3A, 4B, 5A, 7A, and rat PDE9A) or pDEST8 (human PDE1A, 1C, 9A, 10A) and transformed into the Escherichia coli strain DH10Bac (Invitrogen). Bacmid DNA was isolated and purified to obtain baculoviruses coding for each PDE isoform. The baculovirus was amplified by three rounds of transfection into SF9 insect cells. Approximately 72 hours after transfection, once a cytotoxic effect was observed, SF9 cells were centrifuged and frozen in liquid nitrogen, then resuspended in phosphate-buffered saline containing $1 \%$ Triton X-100 with protease inhibitors. After 45 minutes of incubation on ice, cell debris was removed by centrifugation at $13,000 \mathrm{rpm}$ for 30 minutes, and the protein content of the cytoplasmic cell extract containing the recombinant enzymes was determined using a bicinchoninic acid assay kit (Pierce Biotechnology, Rockford, IL).

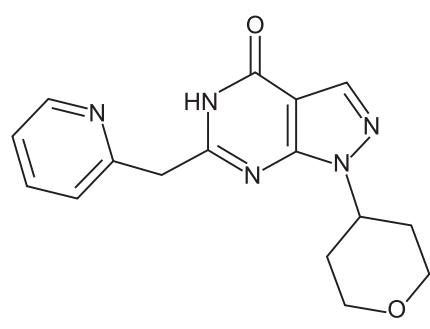

Fig. 1. Structure of BI 409306.

The inhibition of human PDE1A, 1C, 2A, 3A, 4B, 5A, 7A, 9A, and $10 \mathrm{~A}$ and rat PDE9A by BI 409306 was assessed on the basis of the rate of hydrolysis of cGMP and cyclic adenosine monophosphate (cAMP) in the TRKQ7100 PDE $\left[{ }^{3} \mathrm{H}\right] \mathrm{cGMP}$ and TRKQ7090 PDE $\left[{ }^{3} \mathrm{H}\right]$ cAMP scintillation proximity assays (SPA) (GE Healthcare Europe GmbH, Freiburg, Germany). The amount of cell extract containing PDE used in the SPA was adjusted for activity without inhibitors at approximately 1000-2000 counts per minute after 1 hour of incubation at room temperature. This corresponded to total protein concentrations of $480 \mathrm{pg} / \mu \mathrm{l}$ for PDE1A, $2000 \mathrm{pg} / \mu \mathrm{l}$ for PDE1C, 3000 $\mathrm{pg} / \mu \mathrm{l}$ for PDE3A, $624 \mathrm{pg} / \mu \mathrm{l}$ for PDE5A, $160 \mathrm{pg} / \mu \mathrm{l}$ for human PDE9A, $300 \mathrm{pg} / \mu \mathrm{l}$ for PDE2A, 4B, 7A, and $10 \mathrm{~A}$, and $60 \mathrm{pg} / \mu \mathrm{l}$ for rat PDE9A. The reaction mixture contained $20 \mu \mathrm{l}$ of serial dilutions of BI 409396 in an assay buffer containing $0.4 \%$ dimethyl sulfoxide and $10 \mu \mathrm{l}$ of assay buffer containing the diluted PDE protein of interest. Assay buffer contained (in millimolars): Tris ( $\mathrm{pH} 7.4$ adjusted with hydrochloric acid) 50, $\mathrm{MgCl}_{2}$ 8.3, EGTA (pH 7.4 adjusted with $\mathrm{HCl}$ ) 1.7, $0.1 \%$ bovine serum albumin, $0.05 \%$ Tween-20. For the PDE1A and $1 \mathrm{C}$ assays, $200 \mathrm{nM}$ calmodulin and $12 \mathrm{mM} \mathrm{CaCl}_{2}$ were also added. Afterward, $10 \mu \mathrm{l}$ of $\left[{ }^{3} \mathrm{H}\right] \mathrm{cGMP}$ or $\left[{ }^{3} \mathrm{H}\right] \mathrm{cAMP}(80 \mathrm{nM}, 1 \mathrm{mCi} / \mathrm{l}$ in assay buffer) were added, and the reaction mixture was incubated for 1 hour at room temperature before the enzymes were inactivated by addition of $10 \mu \mathrm{l}$ EX00000734 $(50 \mu \mathrm{M})$ in assay buffer and $25 \mu \mathrm{l}$ of yttrium silicate SPA beads $(17.9 \mathrm{mg} / \mathrm{ml})$ in $\mathrm{H}_{2} \mathrm{O}$. The conversion of $\left[{ }^{3} \mathrm{H}\right] \mathrm{cGMP}$ or $\left[{ }^{3} \mathrm{H}\right] \mathrm{cAMP}$ to $\left[{ }^{3} \mathrm{H}\right] \mathrm{GMP}$ or $\left[{ }^{3} \mathrm{H}\right] \mathrm{AMP}$, respectively, was determined using a Topcount NXT scintillation counter (Perkin Elmer, Waltham, MA). All counts obtained in the SPA were subtracted from the counts for the negative control, which contained SF9 cytosolic extract from uninfected cells. Counts were then converted into percentage activity, where the count for a positive control assay, run in the absence of inhibitor, was set at $100 \%$. Percentage activity was used to calculate the BI 409306 concentration at half-maximal inhibition $\left(\mathrm{IC}_{50}\right)$ using GraphPad Prism software.

The selectivity of BI 409306 against PDE6 and other nonPDE targets was evaluated using receptor binding assays according to standard procedure at MDS Pharma Services (Taipei, Taiwan).

\section{Animals and Ethical Considerations}

All animal procedures were performed in accordance with institutional guidelines and European legislation on the use and care of laboratory animals (CEE 86/609 at time of study) and were approved by the ethical committee of the responsible regional council. All efforts were made to minimize animal suffering and to use the minimum number of animals to produce reliable results.

ABBREVIATIONS: BAY 73-6691, 1-(2-chlorophenyl)-6-[(2R)-3,3,3-trifluoro-2-methylpropyl]-1,5-dihydro-4H-pyrazolo[3,4-d]pyrimidine-4-one; CAMP, cyclic adenosine monophosphate; cGMP, cyclic guanosine monophosphate; CSF, cerebrospinal fluid; fEPSPs, field excitatory postsynaptic potentials; IC $\mathrm{I}_{50}$, concentration at half-maximal inhibition; LC-MS/MS, liquid chromatography coupled to mass spectrometry; LTP, long-term potentiation; MK-801, (5R,10S)-(+)-5-methyl-10,11-dihydro-5H-dibenzo[a, $d$ ]cyclohepten-5,10-imine; NMDA, N-methyl-D-aspartate; PDE, phosphodiesterase; PF-04447943, 1,5-dihydro-6-[(3S,4S)-4-methyl-1-(2-pyrimidinylmethyl)-3-pyrrolidinyl]-1-(tetrahydro-2H-pyran-4-yl)$4 H$-pyrazolo[3,4-d]pyrimidin-4-one; SPA, scintillation proximity assays. 


\section{Pharmacokinetic Studies}

Male Wistar rats (Crl:WI[Han]; 200-250 g; Charles River, Sulzfeld, Germany) or male NMRI mice (Crl:NMRI; 20-25 g; Charles River) were orally administered BI $4093061.5 \mathrm{mg} / \mathrm{kg}$. Blood samples were collected at 0.5, 1, 2, and 4 hours and placed in EDTA tubes on ice. Plasma was separated by centrifugation and stored at $-70^{\circ} \mathrm{C}$. In a separate study, Wistar rats were orally administered BI $4093061.5 \mathrm{mg} / \mathrm{kg}$. After 30 minutes, rats were anesthetized with isoflurane and CSF collected using stereotaxic guidance. The CSF samples were stored at $-70^{\circ} \mathrm{C}$. Plasma and CSF concentrations of BI 409306 were determined using liquid chromatography coupled to mass spectrometry (LC-MS/MS).

\section{Microdialysis for Determination of cGMP in the Rat Prefrontal Cortex}

Determination of cGMP levels in the prefrontal cortex of male Sprague-Dawley rats (CD-COBS; 200-250 g; Charles River, Calco, Italy) was performed using transcerebral microdialysis as previously described (Marte et al., 2008). Briefly, microdialysis probes were transversely implanted under chloral hydrate anesthesia $(400 \mathrm{mg} / \mathrm{kg}$; permitted at the time of the study) using the following coordinates: anteroposterior +9.7 , dorsoventral +8.4 from the interaural line (Paxinos and Watson, 1986). Following surgery, rats were allowed to recover for 24 hours. During experiments, the microdialysis probes were infused with artificial cerebrospinal fluid containing (in millimolars): $\mathrm{NaCl} 145, \mathrm{KCl} 3, \mathrm{MgCl}_{2} 1, \mathrm{CaCl}_{2} 1.26, \mathrm{pH} 7.4$, at a flow rate of $5 \mu \mathrm{l} / \mathrm{min}$ Consecutive samples were collected from freely moving rats every 20 minutes for a total of 240 minutes.

Rats received an intraperitoneal injection $(2.5 \mathrm{ml})$ of either BI $409306(1.5,7.5$, or $1 \mathrm{mg} / \mathrm{kg})$ or vehicle. The following were used to prepare $10 \mathrm{ml}$ of vehicle ( $\mathrm{pH} 4.5-4.6): 9 \mathrm{ml}$ of $\mathrm{NaCl} 0.9 \%, 0.67 \mathrm{ml}$ of $\mathrm{HCl}$ $0.1 \mathrm{M}, 0.33 \mathrm{ml}$ of $\mathrm{NaOH} 0.2 \mathrm{M}$. Injections were administered after the collection of the first three basal samples $(0-20,20-40,40-60$ minutes) to estimate baseline cGMP levels. Rats were subsequently euthanized by anesthetic overdose and the probe position verified by optical examination of the fiber tract. Dialysate cGMP content was assayed using a ${ }^{125} \mathrm{I}$-cGMP dual range radioimmunoassay kit (Izotop, Budapest, Hungary) and an acetylation protocol (standard curve range $2-128 \mathrm{fmol}$ ) and a modified bound/unbound separation protocol (Wang et al., 2004).

\section{Determination of cGMP in Rat CSF}

Male Wistar rats (Crl:WI[Han]; 250-320 g; Charles River) 8-10 weeks of age were orally administered BI $409306(0.3,1,3$, or $10 \mathrm{mg} / \mathrm{kg}$ ) or vehicle (sodium chloride solution, $\mathrm{pH} 2.8$ ). After 30 minutes, rats were anesthetized with isoflurane, and CSF samples $(50-100 \mu \mathrm{l})$ were collected through the cisterna magna and centrifuged at $10,000 \mathrm{rpm}$ at $4^{\circ} \mathrm{C}$ for 5 minutes. The concentration of cGMP in rat CSF was determined by highperformance LC-MS/MS on an analytical hydrophilic interaction column with gradient elution. $\left[{ }^{15} \mathrm{~N}_{5}\right]$ cGMP was used as an internal standard. The lower limit of quantification was $0.800 \mathrm{nmol} / \mathrm{l}$ using $20 \mu \mathrm{l}$ of $\mathrm{CSF}$.

\section{Determination of cGMP in Mouse Striatal Tissue}

Adult male CD-1 mice (RjOrl:SWISS; 25-28 g; Janvier Laboratories, Le Genest-St-Isle, France) were intraperitoneally administered BI $409306(0.15$ or $0.5 \mathrm{mg} / \mathrm{kg})$ or vehicle control with and without subcutaneous MK-801 $(0.125 \mathrm{mg} / \mathrm{kg})$. After 30 minutes, animals were euthanized by focused microwave irradiation, and samples of striatal tissue were collected, homogenized, and centrifuged as previously described (Schmidt et al., 2008; Kroker et al., 2014). Supernatant concentration of cGMP was determined by cGMP-ELISA using a commercially available kit (Enzo Life Sciences Inc., Farmingdale, NY) according to the manufacturer's protocol.

\section{Electrophysiological Recording of Hippocampal LTP}

This study was performed at Forschungsinstitut Angewandte Neurowissenschaften GmbH (Magdeburg, Germany).

Hippocampal Slice Preparation. Male Wistar rats (HsdCpb: Wu; Harlan Winkelmann GmbH, Borchen, Germany) 7 to 8 weeks of age were euthanized by a blow to the neck followed by decapitation. The brain was extracted and incised between the hemispheres. The hippocampus was removed, transferred to a cold metal block and mounted onto a tissue chopper. Transverse hippocampal slices of $400 \mu \mathrm{M}$ thickness were prepared at a cutting angle of approximately $70^{\circ}$ to the blade to preserve the laminar structure of the hippocampus.

After preparation, slices were maintained in an interface-type perfusion chamber at a depth of 1-3 $\mathrm{mm}$ beneath the surface of the experimental solution at a constant temperature of $33^{\circ} \mathrm{C}$ for at least 1 hour before recordings were made. Experimental solution was gassed with carbogen $\left(95 \% \mathrm{O}_{2} / 5 \% \mathrm{CO}_{2}\right)$ in a prechamber under mildly hyperbaric conditions (approx. $1 \mathrm{~atm}$ ) and circulated at a flow rate of $2.5 \mathrm{ml}$ per minute. The experimental solution contained (in millimolar): $\mathrm{NaCl} 124, \mathrm{KCl} 4.9, \mathrm{MgSO}_{4} 1.3$, anhydrous $\mathrm{CaCl}_{2} 2.5, \mathrm{KH}_{2} \mathrm{PO}_{4} 1.2$, $\mathrm{NaHCO}_{3} 25.6$, and glucose 10 . The osmolarity and $\mathrm{pH}$ of the solution were corrected to $330 \mathrm{mOsm}$ and $\mathrm{pH} 7.4$, respectively.

Recording. Hippocampal LTP recordings were performed as previously described (Rönicke et al., 2009). Briefly, recordings of field excitatory postsynaptic potentials (fEPSPs) were obtained from recording electrodes placed in the apical dendritic layer of the stratum radiatum of the CA1 region using a direct current amplifier (Model 1700 Differential AC Amplifier; AM-Systems, Sequim, WA). Recording electrodes were pulled from borosilicate glass (1-5-M $\Omega, 3-20-\mu \mathrm{m}$ tip diameter) and were filled with experimental solution. Field potentials were filtered using a low pass filter $(5 \mathrm{kHz})$.

The Schaffer collateral commissural fibers were locally stimulated (1-5 V) using a varnished steel spring wire monopolar stimulation electrode with 20-150- $\Omega$ impedance (Sprint Metal, Reichshof, Germany) and a constant current biphasic stimulus generator (Model 2100 Isolated Pulse Stimulator; AM-Systems). Test stimuli were adjusted to elicit a population spike of approximately $30 \%$ of maximum amplitude.

Following 60 minutes of baseline recordings, LTP was induced using a weak (four sets of paired pulses applied at 200-millisecond intervals) or a strong (10 four-pulse bursts applied at 200 -millisecond intervals repeated once after 10 minutes) tetanic stimulation paradigm. The duration of each pulse was 0.2 milliseconds, and the interpulse interval within pairs or bursts was 10 milliseconds.

BI $409306(0.1,0.3$, or $1 \mu \mathrm{M})$ or vehicle control (0.01\% dimethyl sulfoxide) was bath-applied from 30 minutes prior to the weak tetanic stimulus until the end of the experiment or from 60 minutes prior to the strong tetanic stimulus until 10 minutes after the final stimulus, to evaluate potential long-lasting effects of BI 409306 on protein synthesis-dependent late-phase LTP induced by the strong tetanic stimulus.

\section{Cognitive Assessments}

The T-maze and object recognition tasks were employed to assess the effect of BI 409306 on working and episodic memory, respectively.

T-Maze Continuous Alternation Task in Mice. The study was performed using male CD-1 mice (RjOrl:SWISS; Janvier Laboratories) 4 to 5 weeks of age at Neurofit S.A.S. (Illkirch, France). Mice were group-housed on a reversed light-dark cycle with food and water available ad libitum. The continuous alternation task was performed using a nontransparent T-maze as previously described (de Bruin et al., 2010). Briefly, the experimental protocol consisted of a single session comprising one forced-choice followed by 14 free-choice trials. During the forced-choice trial, one of the arms (either left or right) was blocked, and mice were allowed to explore the open arm. Mice were then allowed to enter either arm in the 14 free-choice trials. If a mouse entered the same arm on two subsequent trials, an error response was recorded. Each session ended once 14 free-choice trials were completed 
or 10 minutes had elapsed, whichever occurred first. Working memory performance was assessed by the percentage of successful alternation over the 14 free-choice trials.

Mice received oral BI $409306(0.007,0.02,0.05,0.1,0.5$, or $2.5 \mathrm{mg} / \mathrm{kg})$ or vehicle ( $0.5 \%$ Tylose) 60 minutes prior to the T-maze test. MK- 801 $(0.1 \mathrm{mg} / \mathrm{kg}$ ) or vehicle (saline) was administered subcutaneously 30 minutes before the assessment.

Object Recognition Task in Mice. Male C57BL/6J mice (C57BL/6JRj; Janvier Laboratories) 3 to 4 months of age were group-housed under standard light conditions with food and water available ad libitum. The object recognition task was performed as described previously (Dodart et al., 1997). Briefly, during trial 1, mice explored an open field in containing two similar and unfamiliar objects. During trial 2, conducted 24 hours later, mice explored an open field containing a familiar object encountered during trial 1 and a novel object. Exploration time in both trials was 10 minutes, and time spent exploring each object was recorded. Episodic memory performance was assessed by the difference between the time spent exploring the novel object compared with the familiar object.

Mice were administered BI $409306(0.015$ or $0.15 \mathrm{mg} / \mathrm{kg})$ or vehicle (saline) via intraperitoneal injection 30 minutes prior to trial 1.

\section{Statistical Analyses}

Microdialysis data were analyzed using nonparametric KruskalWallis test followed by Steel multiple comparison test using KyPlot 3.0 (Kyenslab Inc., Tokyo, Japan). Levels of cGMP in rat CSF and mouse striatal tissue were analyzed using one-way analysis of variance (ANOVA) and with post-hoc Dunnett tests using GraphPad Prism (GraphPad, San Diego, CA). Recording and analysis of fEPSPs was performed using custom-made software (PWIN; Leibniz Institute for Neurobiology, Magdeburg, Germany). Averaging of fEPSP slopes was performed using locally written macros, and LTP data were analyzed using two-way repeated-measures ANOVA. Performance in cognitive tasks was compared using one-way ANOVA with post-hoc Fisher's least significant difference test for the T-maze task, and post-hoc Bonferroni's test for the object recognition task, using GraphPad Prism.

\section{Results}

Potency and Selectivity of BI 409306 In Vitro. BI 409306 demonstrated both potent and selective inhibition of human PDE9A activity, with a calculated mean (S.D.) IC $_{50}$ of 65 (11) nM (Supplementary Fig. 1; Table 1). The $\mathrm{IC}_{50}$ for inhibition of rat PDE9A was $168 \mathrm{nM}$ (Table 1). $\mathrm{IC}_{50}$ values of 1.45 and $1.17 \mu \mathrm{M}$ were calculated for PDE1A and PDE1C, respectively, and no inhibitory activity was observed for PDE2A, 3A, 4B, 5A, 6AB, 7A, and 10A with BI 409306 up to a concentration of $10 \mu \mathrm{M}$ (Table 1). There was no significant activity of BI 409306 at $10 \mu \mathrm{M}$ against 95 non-PDE targets (Supplementary Table 1).

Pharmacokinetics. Following oral dosing with BI 409306 $1.5 \mathrm{mg} / \mathrm{kg}$, rat pharmacokinetic studies indicated that maximal plasma concentration of BI 409306 was reached after approximately 30 minutes, with mean (S.E.M.) $\mathrm{C}_{\max }$ of 892 (47) $\mathrm{nM}$ (Table 2). In mice, maximal plasma concentration of BI 409306 was reached after approximately 60 minutes, with mean (S.E.M.) $\mathrm{C}_{\max }$ of 2227 (288) nM. The concentration of BI 409306 in CSF, a surrogate for free brain concentration, was approximately $20 \%$ of the plasma concentration.

Effect of BI 409306 on cGMP in Rat Prefrontal Cortex and CSF. Following microdialysis, the mean (S.E.M.) basal extracellular cGMP concentration in the rat prefrontal cortex was $4.08(0.51) \mathrm{fmol} / 100 \mu \mathrm{l}$. At 20 minutes postintraperitoneal injection of BI $4093061.5-15 \mathrm{mg} / \mathrm{kg}$, a significant dose-dependent increase in extracellular cGMP levels was observed $\left(\mathrm{X}^{2}=13.95 ; P<0.01 ; \mathrm{df}=3 ; N=5\right.$ animals per group), which peaked after 20 minutes and gradually returned to control values approximately 100 minutes after the injection (Fig. 2A). At 20 minutes, the mean (S.E.M.) percentage increase in extracellular cGMP levels relative to baseline was $187.9 \%$ (49.2) for BI $4093067.5 \mathrm{mg} / \mathrm{kg}$ and 97.6 (49.2) for BI $40930615 \mathrm{mg} / \mathrm{kg}(P<0.05)$. With BI $40930615 \mathrm{mg} / \mathrm{kg}$, significant increases over control values were also observed 40 and 180 minutes after BI 409306 administration. The $1.5 \mathrm{mg} / \mathrm{kg}$ dose did not significantly increase extracellular cGMP over baseline at any time point.

Furthermore, cGMP levels in rat CSF increased in a dosedependent manner following BI 409306 administration, as determined by high-performance LC-MS/MS. Compared with vehicle control, significant increases in cGMP were induced by BI 4093063 and $10 \mathrm{mg} / \mathrm{kg}(N=2-6$ animals per group; $P<0.001$ ) but not by the lower doses of 0.3 and $1 \mathrm{mg} / \mathrm{kg}$ (Fig. 2B). Mean CSF concentration of BI 40930630 minutes after oral administration of BI $4093060.3 \mathrm{mg} / \mathrm{kg}$ in these rats was $67 \mathrm{nM}$. On the basis of the potency of BI 409306 against the rat PDE9 enzyme, a 50\% increase in CSF cGMP was achieved at a CSF BI 409306 exposure of approximately $40 \%$ of the $\mathrm{IC}_{50}$ against PDE9. This is roughly in line with data from a clinical study measuring cGMP increase in CSF after oral treatment with BI 409306 in healthy male volunteers (Boland et al., 2017).

Effect of BI 409306 on MK-801-Induced Reduction in cGMP Levels in Mouse Striatal Tissue. The amount of cGMP detected per mg of mouse striatum was significantly reduced following the administration of the NMDA receptor antagonist MK-801 $(N=8$ animals per group; $P<0.01$; Fig. 3). This reduction was significantly attenuated by coadministration of BI $4093060.5 \mathrm{mg} / \mathrm{kg}$, with a mean increase in cGMP levels of $41 \%$ compared with MK-801 alone $(N=8$ animals per group; $P<0.01)$. After coadministration of BI $4093060.15 \mathrm{mg} / \mathrm{kg}$, cGMP levels were increased by $31 \%$ compared with MK- 801 alone, but this difference did not reach significance.

TABLE 1

Potency and selectivity of BI 409306 for inhibition of human PDE1A, 1C, 2A, 3A, 4B, 5A, 6AB, 7A, 9A, and 10A, and rat PDE9A determined using an SPA to measure hydrolysis of either cGMP or cAMP

\begin{tabular}{lcccccccccccc}
\hline & \multicolumn{10}{c}{ PDE Isoform } \\
\cline { 2 - 10 } & $1 \mathrm{~A}$ & $1 \mathrm{C}$ & $2 \mathrm{~A}$ & $3 \mathrm{~A}$ & $4 \mathrm{~B}$ & $5 \mathrm{~A}$ & $6 \mathrm{AB}$ & $7 \mathrm{~A}$ & 9A (Human) & 9A (Rat) & 10A \\
\hline cGMP/cAMP $^{\text {cGMP }}$ & cGMP & cGMP & cAMP & cAMP & cGMP & cGMP & cAMP & cGMP & cGMP & cAMP \\
$\mathrm{IC}_{50}, \mu \mathrm{M}$, mean (S.D.) & & 1.45 & $1.17(0.27)^{b}$ & $>10$ & $>10$ & $>10$ & $>10$ & $>10$ & $>10$ & $0.07(0.01)^{c}$ & 0.17 & $>10$ \\
\hline
\end{tabular}

${ }^{a} N=2$ dilutions from one experiment unless otherwise stated.

${ }^{b} N=6$ dilutions from three experiments.

${ }^{c} N=8$ dilutions from four experiments. 
TABLE 2

Plasma concentrations of BI 409306 in rats and mice 0.5, 1, 2, and 4 hours after oral administration of BI $4093061.5 \mathrm{mg} / \mathrm{kg}$

\begin{tabular}{|c|c|c|c|c|}
\hline & \multicolumn{4}{|c|}{ Time (h) } \\
\hline & 0.5 & 1.0 & 2.0 & 4.0 \\
\hline \multicolumn{5}{|c|}{ Mean $^{a}$ (S.E.M.) plasma concentration $[\mathrm{nM}]$} \\
\hline Rat & $892(47)$ & $714(146)$ & $683(129)$ & $50(15)$ \\
\hline Mouse & $2097(215)$ & 2227 (288) & $1042(262)$ & $183(62)$ \\
\hline
\end{tabular}

${ }^{a} N=3$ animals for each experiment.

Effect of BI 409306 on Hippocampal LTP Ex Vivo. When BI 409306 was applied 30 minutes before a weak tetanic stimulation, it significantly enhanced LTP at $1 \mu \mathrm{M}$ compared with vehicle control (vehicle control, $N=12$ slices from six animals; BI 409306, $N=9$ slices from five animals; $P=0.021$ ), but not at 0.1 or $0.3 \mu \mathrm{M}$ (Fig. $4 \mathrm{~A}$ ). When applied 60 minutes before a strong stimulus, BI 409306 significantly enhanced LTP compared with vehicle control at $0.3 \mu \mathrm{M}$ (vehicle control, $N=10$ slices from five animals; BI 409306, $N=10$ slices from five animals; $P=0.048$ ), but not at 1 or $0.1 \mu \mathrm{M}$ (Fig. 4B).

Effect of BI 409306 on Working and Episodic Memory. In the T-maze task, administration of MK- $8010.1 \mathrm{mg} / \mathrm{kg}$, led to a significant reduction in spontaneous alternation (i.e., the percentage of trials in which correct alternation was observed) from a mean (S.D.) of $71.4 \%$ (2.1) observed with vehicle to $44.3 \%$ (2.1), approximately chance level (50\% correct alternation), with MK-801 $(P<0.001)$. Oral treatment of mice with BI 409306 at all doses tested $(0.007-2.5 \mathrm{mg} / \mathrm{kg})$ led to an attenuation or reversal of the MK-801-induced reduction in spontaneous alternation compared with the MK-801-treated group ( $N=10$ animals per group; $P<0.05$; Fig. 5A). On the basis of the mean plasma concentration of BI 409306 measured in mice 1 hour after administration of BI $4093061.5 \mathrm{mg} / \mathrm{kg}$, the extrapolated plasma exposure levels of mice in the T-maze at most effective doses $(0.02-0.5$ $\mathrm{mg} / \mathrm{kg}$ intraperitoneal) would be in the range of $67-1675 \mathrm{nM}$. These extrapolated plasma exposures correspond to BI 409306 concentrations in CSF of $13-335 \mathrm{nM}$, which is approximately 0.1- to 2-fold of the PDE9A IC $\mathrm{I}_{50}$ on the basis of potency against the rat enzyme. Since the $\mathrm{IC}_{50}$ value for inhibition of PDE1

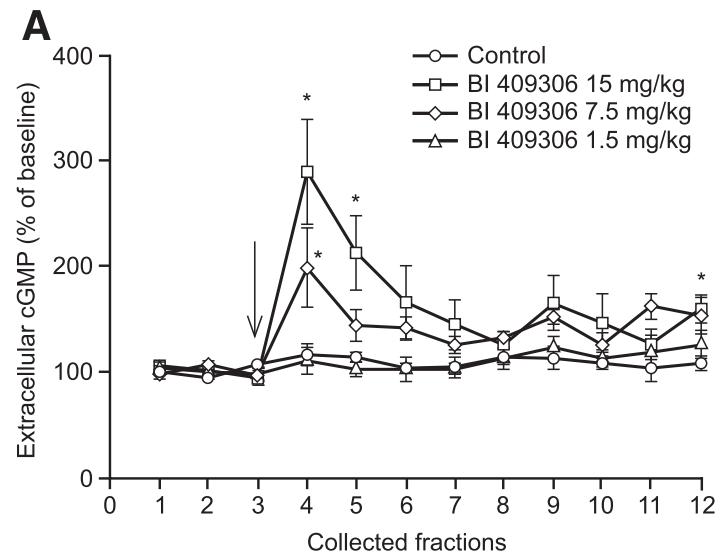

isoforms by BI 409306 is $>1 \mu \mathrm{M}$, the memory-improving efficacy of BI 409306 can be attributed solely to the inhibition of PDE9.

In the object recognition task, during trial 1 exploration times for the two objects did not differ between mice treated with vehicle or BI 409306 at either 0.015 or $0.15 \mathrm{mg} / \mathrm{kg}$ (data not shown). At trial 2, which was performed 24 hours after trial 1, animals treated with BI $4093060.15 \mathrm{mg} / \mathrm{kg}$ spent significantly more time exploring the novel object compared with the familiar object $(P<0.001)$, whereas there was no significant difference between time spent exploring the novel and the familiar object in mice treated with vehicle or BI $4093060.015 \mathrm{mg} / \mathrm{kg}$ (Fig. 5B).

\section{Discussion}

This study assessed the in vitro potency and selectivity of BI 409306 and characterized its effects on synaptic plasticity and cognitive performance in rodents. Enzymatic and receptor binding assays demonstrated that $\mathrm{BI} 409306$ is a potent and selective inhibitor of human PDE9A $\left(\mathrm{IC}_{50}=65 \mathrm{nM}\right)$, with weak affinity for PDE1 (approx. 20-fold selectivity) and $>100$ fold selectivity for PDE9A over other PDE enzymes as well as a range of non-PDE targets. Systemic administration of BI 409306 led to an increase in cGMP levels in the rat prefrontal cortex and CSF, and to a reversal of cGMP reduction induced by the NMDA receptor antagonist MK-801 in mouse striatal tissue. These observations suggest that BI 409306 binds to and inhibits PDE9A in the brain in vivo, showing functional target engagement. Furthermore, this study demonstrated for the first time that NMDA receptor blockade leads to a decrease

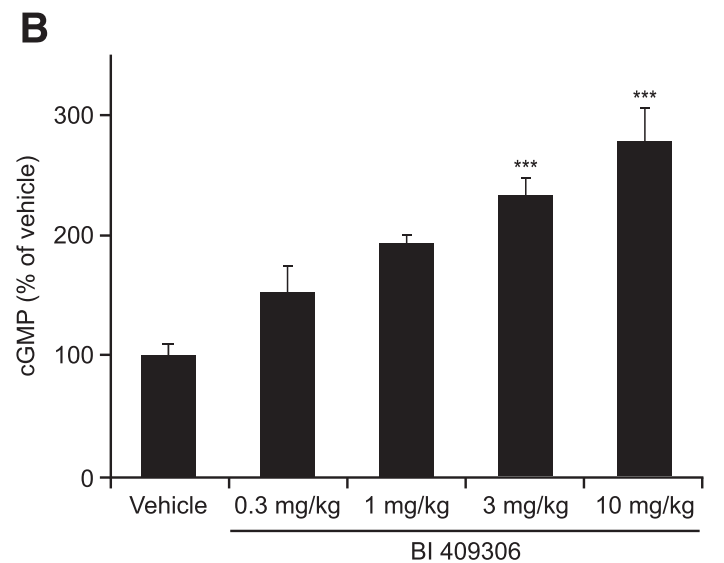

Fig. 2. The effect of BI 409306 on cGMP levels in (A) rat prefrontal cortex and (B) CSF. (A) BI 409306 1.5, 7.5, or 15 mg/kg was administered intraperitoneally (arrow). Microdialysis fractions were collected every 20 minutes. Data are expressed as mean ( \pm S.E.M.) percentages of the mean basal level of extracellular cGMP (defined as 100\%), which was determined by averaging the content of the first three samples collected before BI 409306 administration. $N=5$ animals per group. (B) BI $4093060.3,1,3$, or $10 \mathrm{mg} / \mathrm{kg}$ was administered orally, and CSF was collected $30 \mathrm{minutes}$ later. Data are expressed as mean ( \pm S.E.M.) percentage of cGMP levels after administration of vehicle control (defined as $100 \%$ ). $N=2-6$ animals per group. $* P<0.05$, $* * * P<0.001$ compared with control group. 




Fig. 3. The effect of BI 409306 on MK-801-induced reduction in cGMP levels in mouse striatal tissue. BI 4093060.15 or $0.5 \mathrm{mg} / \mathrm{kg}$ was administered intraperitoneally. MK-801 $0.125 \mathrm{mg} / \mathrm{kg}$ was administered subcutaneously, and tissue was collected 30 minutes later. Data are presented as mean ( \pm S.E.M.). $N=8$ animals per group. ${ }^{*} P<0.05$, ** $P<0.01$ compared with MK-801 group.

in cGMP levels that could be reversed by PDE9A inhibition. This supports the hypothesis that PDE9A is located downstream of NMDA receptor activation and acts as a regulator of the cGMP-related postsynaptic signaling cascade, which is involved in synaptic plasticity. Indeed, application of BI 409306 facilitates synaptic plasticity in hippocampal slices as demonstrated by enhanced LTP induced by both weak and strong tetanic stimulation. This suggests an enhancement of both early and late phases of LTP, which are independent of and dependent on protein synthesis, respectively. Finally, mice treated with BI 409306 demonstrated improved performance on T-maze and object recognition tasks, which assess working and episodic memory, respectively.

In the first-in-human study, BI 409306 demonstrated good safety and tolerability following the administration of single rising doses up to $350 \mathrm{mg}$ in healthy male participants, and BI 409306 was rapidly absorbed and eliminated (Moschetti et al., 2016). BI 409306 was also well tolerated over 14 days of daily treatment in healthy individuals (Moschetti et al., 2018) and in patients with schizophrenia (Brown et al., 2018). However, to fully assess the efficacy of BI 409306 in human patients, translatable biomarkers are required that provide similar objective measures of drug outcomes in patients, healthy volunteers, and animal models. In the present study, levels of cGMP in the rat prefrontal cortex reflected cGMP levels in rat CSF following administration of BI 409306. This is consistent with previous studies showing increases in cGMP levels following PDE9A inhibition in rodent CSF (Hutson et al., 2011; Kleiman et al., 2012) and in mouse hippocampus (Kroker et al., 2014). This suggests that cGMP levels in CSF might be a suitable biomarker for central inhibition of PDE9A in human patients. In support of this, a phase I proof-ofmechanism study of BI 409306 (25-, 50-, 100-, or 200-mg single dose) in healthy males demonstrated dose-dependent increases in CSF cGMP that reflected plasma and CSF drug exposure (Boland et al., 2017). Overall, these studies support the utility of CSF cGMP levels as a translatable biomarker of functional target engagement between animal and human studies.

Previous studies investigating the effects of PDE9A inhibitors on LTP had findings similar to those observed in this study. For example, the PDE9A inhibitor BAY 73-6691 enhanced early LTP induced by weak theta stimulation in rat hippocampal slices (van der Staay et al., 2008) and protein synthesis-dependent late LTP induced by spaced high-frequency $(100-\mathrm{Hz})$ tetanic stimulation in mouse hippocampal slices (Kroker et al., 2012). PF-04447943, another PDE9A inhibitor, promoted LTP induced by a weak but not strong tetanic stimulation in ex vivo mouse hippocampus (Hutson et al., 2011). In the present study, BI 409306 enhanced LTP induced with both weak and strong tetanic stimulation, suggesting that BI 409306 can promote both early- and late-phase LTP. In addition, given that BI 409306 reversed the MK-801-induced decrease in brain cGMP levels, BI 409306 may enhance hippocampal LTP by increasing cGMP levels and promoting NMDA receptor signaling even under conditions of compromised NMDA receptor function or signaling, which has been suggested as playing a role in neuropsychiatric diseases such as schizophrenia (Lin et al., 2012). However, it should be noted that the enhancement of LTP induced with weak and strong tetanic stimulation was only observed at BI 409306 concentrations of 1 and $0.3 \mu \mathrm{M}$, respectively, which may indicate a bell-shaped dose-response curve, and thus presumably a narrow window of efficacy.

In previous studies assessing the effects of PDE9A inhibition on learning and memory, BAY73-6691 improved performance in object recognition and social recognition tasks, reversed an MK-801-induced working memory deficit, and reversed a memory deficit in a passive avoidance task induced by the muscarinic antagonist scopolamine (van der Staay et al., 2008). In addition, we have shown previously that BAY73-6691 attenuated deficits in an object location memory task in amyloid precursor protein-transgenic Tg2576 mice exhibiting Alzheimer's disease pathology (Kroker et al., 2014). Another compound, PF-04447943, also showed a reversal of working memory deficits induced by the NMDA receptor antagonist ketamine, and of deficits in episodic and spatial memory induced by the muscarinic antagonist scopolamine (Kleiman et al., 2012). In the present study, animals treated with BI 409306 showed significant reversal of an MK801-induced deficit in spontaneous alternation, indicating improved working memory performance. In addition, BI 409306 also improved episodic memory performance, demonstrated by an object recognition task with a 24-hour delay between trials 1 and 2 . At trial 2, animals treated with BI $4093060.15 \mathrm{mg} / \mathrm{kg}$ spent significantly more time exploring the novel object compared with the familiar object. This indicates that animals treated with BI $4093060.15 \mathrm{mg} / \mathrm{kg}$ remembered the object presented at trial 1 , in contrast to animals treated with vehicle or $0.015 \mathrm{mg} / \mathrm{kg}$. Notably, BI 409306 was administered only at memory acquisition phase, 24 hours prior to the trial in which improvements in memory retrieval were observed. This suggests that BI 409306 may promote consolidation of short- to long-term memory following the administration of a single dose as was also suggested by studies of other PDE inhibitors (Heckman et al., 2018). In existing literature, a distinction is drawn between mechanisms underlying short-term and long-term memory; the former has been suggested as reflecting the early phase of LTP, whereas the latter might reflect the long-lasting late phase of LTP (Reneerkens et al., 2009). Presynaptic cGMP signaling is thought to support early LTP and to activate the conversion of early LTP to late LTP through a cyclic 
A
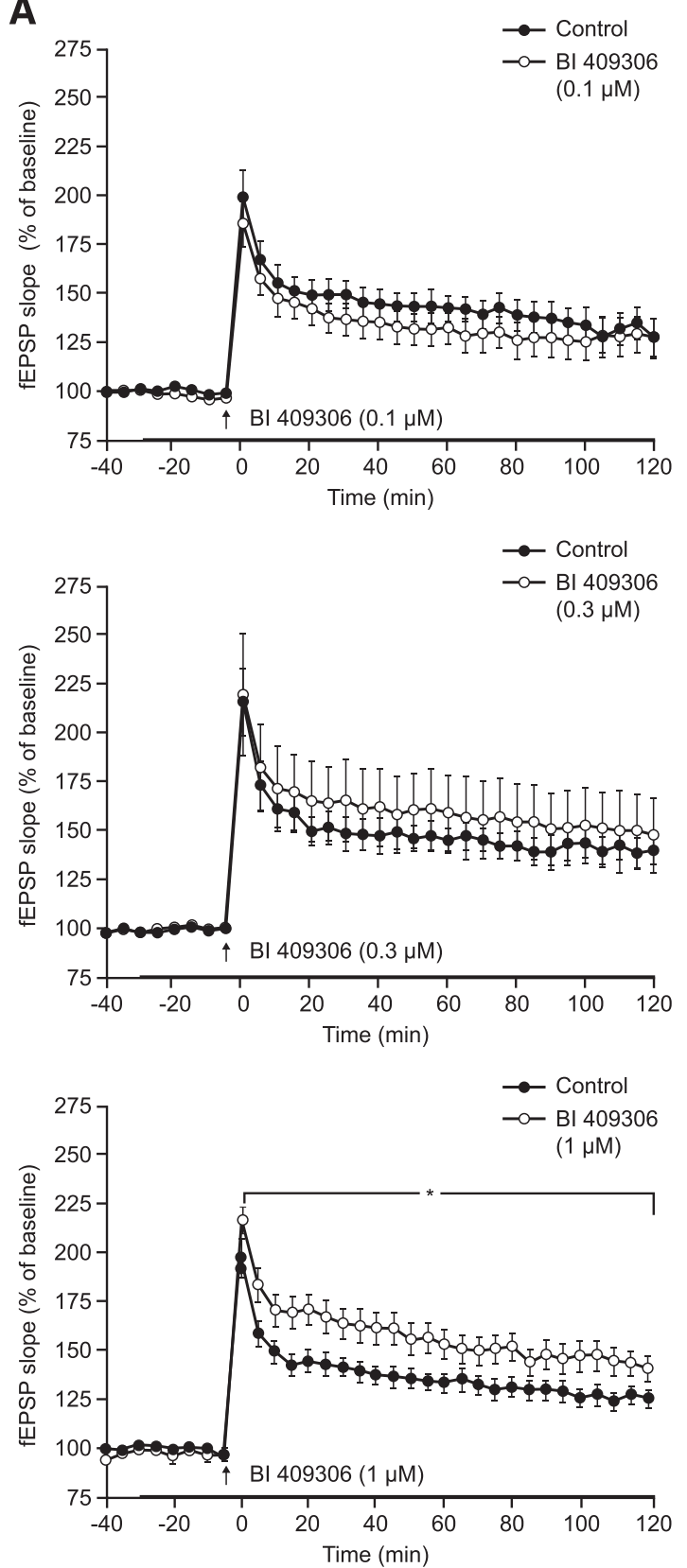

B
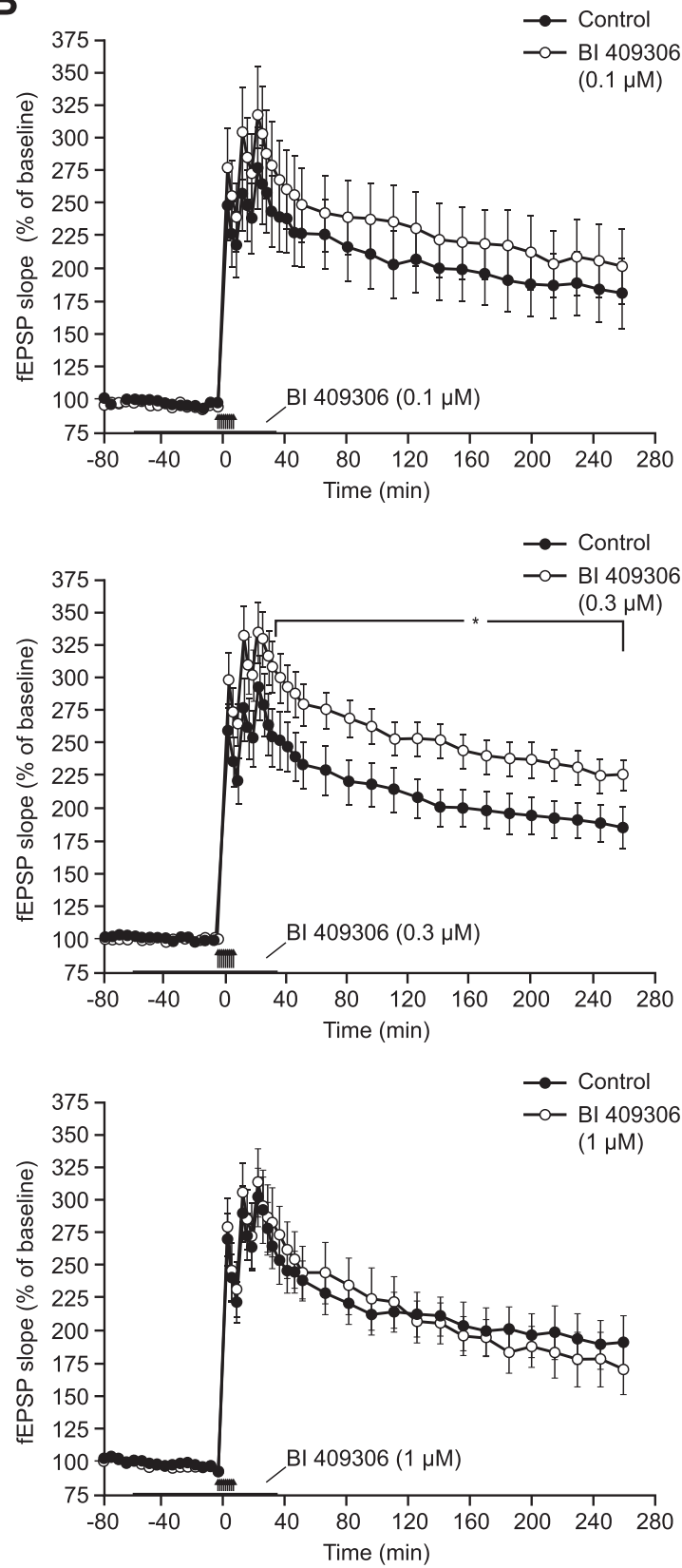

Fig. 4. The effect of BI 409306 on LTP in ex vivo rat hippocampus induced by (A) weak and (B) strong tetanic stimulation of Schaffer collateralcommissural fibers in the stratum radiatum of the CA1 region. BI $4093060.1,0.3$, or $1 \mu \mathrm{M}$ was bath-applied during the period indicated on the $\mathrm{x}$-axis. Arrows indicate the onset of the weak or strong tetanic stimulus. Data are expressed as mean ( \pm S.E.M. $)$ percentage of average fEPSP slope at baseline (defined as 100\%). $N=3-12$ slices from three to six animals per group. ${ }^{*} P<0.05$ for effect of treatment compared with control group.

adenosine monophosphate-dependent pathway (Ricciarelli and Fedele, 2018). Conversion of early LTP signaling to late LTP supported by cGMP might explain the improvement in episodic memory performance 24 hours after a single dose of BI 409306 in the present study. This might also indicate that BI 409306 improves long-term memory performance through cGMP-mediated promotion of synaptic plasticity, including structural changes such as enhanced development of dendritic spines and synapses, which has been demonstrated in cultured hippocampal neurons and in cortical pyramidal neurons in mice following PDE9A inhibition (Hutson et al., 2011; Lai et al., 2018).

The effects of BI 409306 on cognition have also been explored in a double-blind, randomized phase II study
(NCT02281773) comparing BI 409306 at doses up to $100 \mathrm{mg}$ and placebo in patients with schizophrenia. No significant differences were observed on a range of cognitive outcomes, and the primary endpoints were not met after 12 weeks of treatment (Brown et al., 2019). Two double-blind, randomized phase II studies (NCT02240693 and NCT02337907) have also been conducted in 128 and 329 patients with prodromal and mild Alzheimer's disease, respectively. These studies demonstrated no significant effect of BI 409306 compared with placebo on the primary efficacy endpoint of Neuropsychological Test Battery total z-score after 12 weeks of treatment (Frölich et al., 2019). Furthermore, in a phase I study that included measures of verbal learning and visuospatial memory 
A

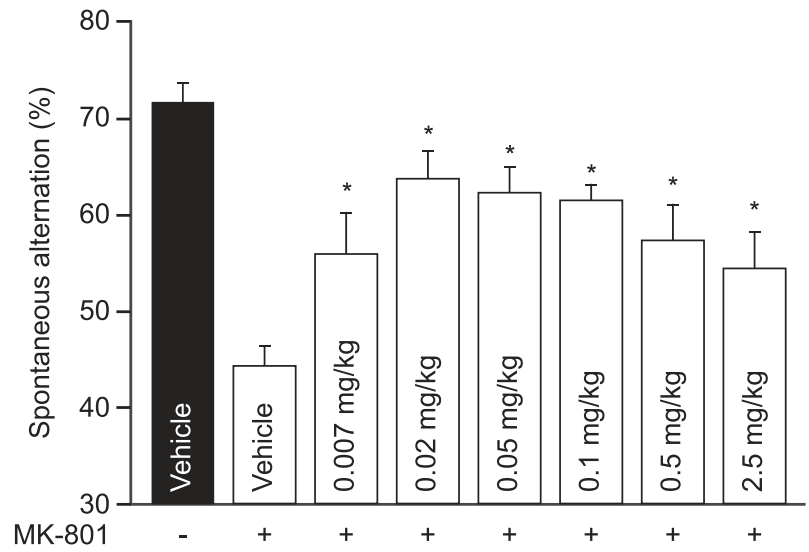

B

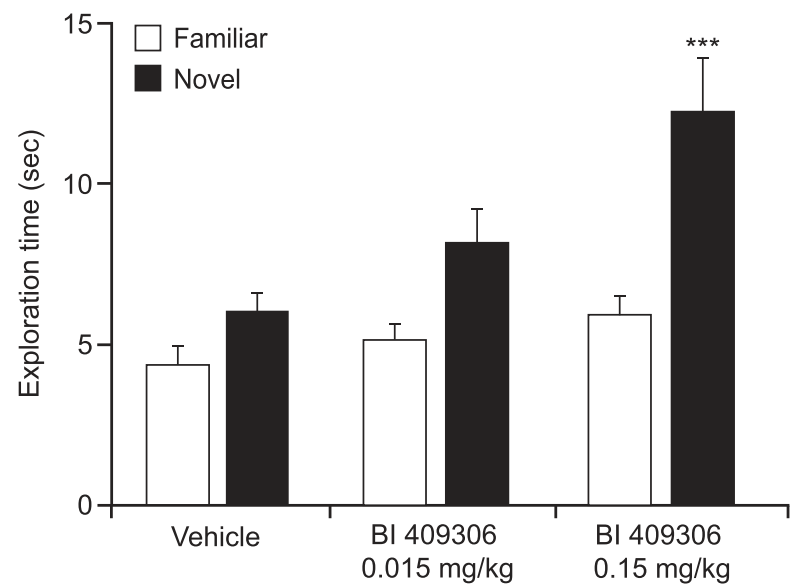

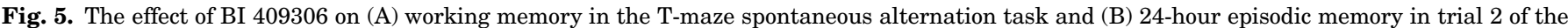

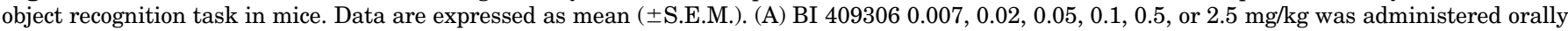

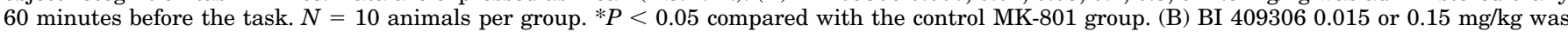
administered intraperitoneally 30 minutes before trial $1 . N=10-11$ animals per group. $* * * P<0.001$ compared with the familiar object.

as secondary endpoints, no significant treatment effects of BI 409306 on cognitive outcomes were observed in 38 patients with schizophrenia (Brown et al., 2018). Overall, the observed effects of BI 409306 on cognition in patients contrast with the effects in rodents reported in the present study. This may indicate that the effects of BI 409306 in patients require further investigation. For example, BI 409306 may have beneficial effects on cognition when administered at a higher dose, over a longer treatment period, or in patients with different stages of schizophrenia or Alzheimer's disease (Brown et al., 2019; Frölich et al., 2019). Alternatively, it may be that drugs enhancing synaptic plasticity are more effective in the treatment of other indications, or that the effects of such drugs observed in the rodent models used in the present study translate poorly to cognitive improvement in patients with schizophrenia and Alzheimer's disease.

In conclusion, these data suggest that PDE9A inhibition with BI 409306 increases cGMP levels in the brain, promotes both early and late LTP, and improves performance on cognitive tasks assessing working and episodic memory in rodents.

\section{Acknowledgments}

We thank Dr. E. Andriambeloson from Neurofit S.A.S. for his support regarding the T-maze study, as well as N. Kötteritzsch, S. Schwaeble, T. Sauter, S. Landthaler, K. Böhm, and R. Beilmann for their technical assistance. Editorial support in the form of initial preparation of the outline on the basis of input from all authors, and collation and incorporation of author feedback to develop subsequent drafts, assembling tables and figures, copyediting, and referencing was provided by Mark Condon, DPhil, of Fishawack Communications Ltd, UK, and was funded by Boehringer Ingelheim International $\mathrm{GmbH}$.

\section{Authorship Contributions}

Participated in research design: Rosenbrock, Giovannini, Schänzle, Koros, Fuchs, Marti, Reymann, Schröder, Fedele, Dorner-Ciossek.

Conducted experiments: Rosenbrock, Schänzle, Fuchs, Marti, Schröder, Fedele, Dorner-Ciossek.

Contributed new reagents or analytic tools: Runge.

Performed data analysis: Rosenbrock, Giovannini, Schänzle, Koros, Runge, Fuchs, Marti, Schröder, Fedele, Dorner-Ciossek.
Wrote or contributed to the writing of the manuscript: All authors.

\section{References}

Andreeva SG, Dikkes P, Epstein PM, and Rosenberg PA (2001) Expression of cGMPspecific phosphodiesterase 9A mRNA in the rat brain. J Neurosci 21:9068-9076.

Arancio O, Kandel ER, and Hawkins RD (1995) Activity-dependent long-term enhancement of transmitter release by presynaptic $3^{\prime}, 5^{\prime}$-cyclic GMP in cultured hippocampal neurons. Nature 376:74-80.

Boland K, Moschetti V, Dansirikul C, Pichereau S, Gheyle L, Runge F, ZimdahlGelling H, and Sand M (2017) A phase I, randomized, proof-of-clinical-mechanism study assessing the pharmacokinetics and pharmacodynamics of the oral PDE9A inhibitor BI 409306 in healthy male volunteers. Hum Psychopharmacol 32:e2569.

Brown D, Daniels K, Pichereau S, and Sand M (2018) A phase Ic study evaluating the safety, tolerability, pharmacokinetics, and cognitive outcomes of BI 409306 in patients with mild-to-moderate schizophrenia. Neurol Ther 7:129-139

Brown D, Nakagome K, Cordes J, Brenner R, Gründer G, Keefe RSE, Riesenberg R, Walling DP, Daniels K, Wang L, et al. (2019) Evaluation of the efficacy, safety, and tolerability of BI 409306, a novel phosphodiesterase 9 inhibitor, in cognitive impairment in schizophrenia: a randomized, double-blind, placebo-controlled, phase II trial. Schizophr Bull 45:350-359.

de Bruin NM, Prickaerts J, Lange JH, Akkerman S, Andriambeloson E, de Haan M, Wijnen J, van Drimmelen M, Hissink E, Heijink L, et al. (2010) SLV330, a cannabinoid CB1 receptor antagonist, ameliorates deficits in the T-maze, object recognition and Social Recognition Tasks in rodents. Neurobiol Learn Mem 93: $522-531$.

Dodart JC, Mathis C, and Ungerer A (1997) Scopolamine-induced deficits in a twotrial object recognition task in mice. Neuroreport 8:1173-1178.

Dorner-Ciossek C, Kroker KS, and Rosenbrock H (2017) Role of PDE9 in cognition. Adv Neurobiol 17:231-254.

Fisher DA, Smith JF, Pillar JS, St Denis SH, and Cheng JB (1998) Isolation and characterization of PDE9A, a novel human cGMP-specific phosphodiesterase. $J$ Biol Chem 273:15559-15564.

Frölich L, Wunderlich G, Thamer C, Roehrle M, Garcia M Jr, and Dubois B (2019) Evaluation of the efficacy, safety and tolerability of orally administered BI 409306, a novel phosphodiesterase type 9 inhibitor, in two randomised controlled phase II studies in patients with prodromal and mild Alzheimer's disease. Alzheimers Res Ther 11:18.

Heckman PRA, Blokland A, Bollen EPP, and Prickaerts J (2018) Phosphodiesterase inhibition and modulation of corticostriatal and hippocampal circuits: clinical overview and translational considerations. Neurosci Biobehav Rev 87:233-254.

Hutson PH, Finger EN, Magliaro BC, Smith SM, Converso A, Sanderson PE, Mullin D, Hyde LA, Eschle BK, Turnbull Z, et al. (2011) The selective phosphodiesterase 9 (PDE9) inhibitor PF-04447943 (6-[(3S,4S)-4-methyl-1-(pyrimidin-2-ylmethyl)pyrrolidin-3-yl]-1-(tetrahydro-2H-pyran-4-yl)-1,5-dihydro-4H-pyrazolo[3,4-d]pyrimidin4-one) enhances synaptic plasticity and cognitive function in rodents. Neuropharmacology 61:665-676.

Kleiman RJ, Chapin DS, Christoffersen C, Freeman J, Fonseca KR, Geoghegan KF, Grimwood S, Guanowsky V, Hajós M, Harms JF, et al. (2012) Phosphodiesterase 9A regulates central cGMP and modulates responses to cholinergic and monoaminergic perturbation in vivo. J Pharmacol Exp Ther 341:396-409.

Kroker KS, Mathis C, Marti A, Cassel JC, Rosenbrock H, and Dorner-Ciossek C (2014) PDE9A inhibition rescues amyloid beta-induced deficits in synaptic plasticity and cognition. Neurobiol Aging 35:2072-2078.

Kroker KS, Rast G, Giovannini R, Marti A, Dorner-Ciossek C, and Rosenbrock H (2012) Inhibition of acetylcholinesterase and phosphodiesterase-9A has differential effects on hippocampal early and late LTP. Neuropharmacology 62:1964-1974. 
Lai B, Li M, Hu W, Li W, and Gan WB (2018) The phosphodiesterase 9 inhibitor pf04449613 promotes dendritic spine formation and performance improvement after motor learning. Dev Neurobiol 78:859-872.

Lee YS and Silva AJ (2009) The molecular and cellular biology of enhanced cognition. Nat Rev Neurosci 10:126-140.

Lin CH, Lane HY, and Tsai GE (2012) Glutamate signaling in the pathophysiology and therapy of schizophrenia. Pharmacol Biochem Behav 100:665-677.

Lu YF, Kandel ER, and Hawkins RD (1999) Nitric oxide signaling contributes to late-phase LTP and CREB phosphorylation in the hippocampus. J Neurosci 19:10250-10261.

Marte A, Pepicelli O, Cavallero A, Raiteri M, and Fedele E (2008) In vivo effects of phosphodiesterase inhibition on basal cyclic guanosine monophosphate levels in the prefrontal cortex, hippocampus and cerebellum of freely moving rats. J Neurosci Res 86:3338-3347.

Morris RG (2013) NMDA receptors and memory encoding. Neuropharmacology 74:32-40

Moschetti V, Boland K, Feifel U, Hoch A, Zimdahl-Gelling H, and Sand M (2016) First-in-human study assessing safety, tolerability and pharmacokinetics of $\mathrm{BI}$ 409306, a selective phosphodiesterase 9A inhibitor, in healthy males. $\mathrm{Br} \mathrm{J} \mathrm{Clin}$ Pharmacol 82:1315-1324.

Moschetti V, Kim M, Sand M, Wunderlich G, Andersen G, Feifel U, Jang IJ, Timmer W, Rosenbrock H, and Boland $\mathrm{K}$ (2018) The safety, tolerability and pharmacokinetics of BI 409306, a novel and potent PDE9 inhibitor: overview of three phase I randomised trials in healthy volunteers. Eur Neuropsychopharmacol 28:643-655.

Paxinos G and Watson C (1986) The Rat Brain in Stereotaxic Coordinates, Academic Press, San Diego, CA.

Reneerkens OA, Rutten K, Steinbusch HW, Blokland A, and Prickaerts J (2009) Selective phosphodiesterase inhibitors: a promising target for cognition enhancement. Psychopharmacology (Berl) 202:419-443.
Ricciarelli R and Fedele E (2018) cAMP, cGMP and amyloid $\beta$ : three ideal partners for memory formation. Trends Neurosci 41:255-266.

Rönicke R, Schröder UH, Böhm K, and Reymann KG (2009) The Na+/H+ exchanger modulates long-term potentiation in rat hippocampal slices. Naunyn Schmiedebergs Arch Pharmacol 379:233-239.

Schmidt CJ, Chapin DS, Cianfrogna J, Corman ML, Hajos M, Harms JF, Hoffman WE, Lebel LA, McCarthy SA, Nelson FR, et al. (2008) Preclinical characterization of selective phosphodiesterase 10A inhibitors: a new therapeutic approach to the treatment of schizophrenia. J Pharmacol Exp Ther 325:681-690.

Taqatqeh F, Mergia E, Neitz A, Eysel UT, Koesling D, and Mittmann T (2009) More than a retrograde messenger: nitric oxide needs two cGMP pathways to induce hippocampal long-term potentiation. J Neurosci 29:9344-9350.

van der Staay FJ, Rutten K, Bärfacker L, Devry J, Erb C, Heckroth H, Karthaus D, Tersteegen A, van Kampen M, Blokland A, et al. (2008) The novel selective PDE9 inhibitor BAY 73-6691 improves learning and memory in rodents. Neuropharmacology 55:908-918.

Volianskis A, France G, Jensen MS, Bortolotto ZA, Jane DE, and Collingridge GL (2015) Long-term potentiation and the role of $\mathrm{N}$-methyl-D-aspartate receptors. Brain Res 1621:5-16.

Wang M, Urenjak J, Fedele E, and Obrenovitch TP (2004) Effects of phosphodiesterase inhibition on cortical spreading depression and associated changes in extracellular cyclic GMP. Biochem Pharmacol 67:1619-1627.

Address correspondence to: Dr. Holger Rosenbrock, Boehringer Ingelheim International GmbH, Birkendorfer Str. 65, 88397 Biberach an der Riß, Germany. E-mail: holger.rosenbrock@boehringer-ingelheim.com 